\title{
La modernité à l'épreuve de la peinture : Une approche du sens critique des derniers tableaux de Malévitch
}

Par ARTHUR COOLS

Universiteit Antwerpen

\section{Introduction}

Les réflexions élaborées dans cet article se rapportent à la dernière période (1928-1932) de Kazimir Malévitch, devenu célèbre par le «Carré noir » (1914) qui a inauguré le suprématisme. En dépit de l'ouverture vers l'abstraction, caractéristique du suprématisme, on constate dans les derniers tableaux un retour au figuratif. Les figures sont certes très réduites, mais tout de même reconnaissables comme figures humaines ou têtes d'homme. Le titre des tableaux leur prête d'ailleurs un nom: " paysans », "femme rouge », «figure ». Le visage n'est pas marqué et, si les yeux regardent, ils restent abstraits. On retrouve en outre dans certains tableaux un horizontal qui marque l'ouverture d'un horizon et un rapport au monde. Le paysage est parfois divisé en des champs de couleurs diverses, le ciel en fumées de couleurs grises et noires. Les couleurs sont fortes, créant des oppositions violentes. La division des champs est floue ; les couleurs semblent flotter. Le rapport entre figure et monde est détaché : ainsi, par exemple, la femme rouge nous donne l'impression de s'élever au-dessus du paysage ou encore, dans le tableau «Paysans », la figure centrale derrière laquelle on peut reconnaître des avions au-dessus des champs au lointain est comme détachée du paysage.

Comment interpréter ces tableaux ? On a suggéré que Malévitch avait voulu s'adapter aux exigences esthétiques du communisme staliniste de son temps - sans succès, il faut le dire, car son œuvre fut mise sous surveillance et exclue du canon officiel de l'esthétique communiste. Dans une telle 
optique, le retour au figuratif resterait en-deçà de la percée réalisée par le suprématisme et signifierait un retour en arrière, une sorte de trahison à l'égard de sa position avant-gardiste d'avant la révolution russe. Cela expliquerait pourquoi ces tableaux n'ont pas attiré de commentaires, à l'encontre du « Carré noir », qui est peut-être un des tableaux les plus commentés par les philosophes dans l'histoire de la philosophie de l'art ${ }^{1}$.

On connait les interprétations que le suprématisme de Malévitch a suscitées dans la théorie de l'art qui veut tenir compte de la percée de l'avant-garde. Selon Jean-François Lyotard, l'œuvre du peintre russe reste moderne, témoignant d'un rapport mélancolique à l'égard de la perte de la présence de la figuration, et en ce sens précis elle ne relève pas encore de l'esprit d'innovation caractéristique du postmoderne, comme c'est le cas pour Duchamp ${ }^{2}$. Pour Thierry de Duve, qui ne voit aucune différence essentielle entre les ready made de Duchamp et les aboutissements du suprématisme, Malévitch touche à sa façon à la fin de la peinture ${ }^{3}$. Selon cette ligne interprétative, le retour au figuratif dans la dernière période n'a retenu aucune attention particulière. Pour Lyotard, ce retour corrobore sans doute la visée profondément mystique et mélancolique de Malévitch. Pour de Duve, ce même retour est dépourvu d'un sens historique important.

Cet article tentera une autre approche. Loin de considérer le retour au figuratif dans ces tableaux comme un retour en arrière et une rupture avec les acquis du suprématisme, je voudrais soutenir l'hypothèse qu'il signale au contraire une prise de conscience de la nécessité d'approfondir et de renouveler les pouvoirs critiques inhérents au suprématisme dans un nouveau contexte idéologique - post-révolutionnaire. Dans une telle perspective, la recherche du dernier Malévitch devient un laboratoire pictural pour étudier les conditions du sens et du pouvoir critiques de la figuration en peinture, faisant preuve d'une vigilance à l'égard du présent. Je voudrais donc présenter Malévitch comme «Kulturkritiker » et l'image picturale comme un espace qui examine les moyens et les limites qui lui sont propres afin de pouvoir réfléchir la crise de la modernité. Il ne s'agit pas, par là, de renouer subrepticement avec une tradition classique de la représentation (entendue ici

${ }^{1}$ Pour une mise au point, voir e.a. Felix Philipp Ingold, «Welt und Bild. Zur Begründung der suprematistischen Ästhetik bei Kazimir Malevič », dans Gottfried Boehm (Hrsg.), Was ist ein Bild ?, München, Fink, 1995, p. 367-410.

2 Jean-François Lyotard, Le Postmoderne expliqué aux enfants. Correspondance 1982-1985, Paris, Éditions Galilée, 1986, p. 30.

${ }^{3}$ Thierry de Duve, « Malévitch et la question de l'abandon », dans Id., Nominalisme pictural. Marcel Duchamp : La peinture et la modernité, Paris, Éditions de Minuit, 1984, p. 227-233. 
comme art figuratif ou figuration picturale), mais d'examiner par quels sens la notion de représentation en peinture peut être enrichie malgré toutes les critiques qu'elle a subi au $\mathrm{XX}^{\mathrm{e}}$ siècle. À quel sens de la représentation invite le retour au figuratif dans les derniers tableaux de Malévitch ?

Entre le «Carré noir » et le suprématisme qu'il inaugure, d'une part, et le retour au figuratif à la fin de sa vie, de l'autre, il semble que le rapport de Malévitch à la modernité ait changé. Ce changement concerne l'essence et la tâche de la peinture dans les péripéties de la modernité qui, selon une opinion très répandue, a causé le déclin de l'image picturale au profit de l'image photographique et cinématographique en vertu de l'application des nouvelles technologies dans la production artistique. Ce changement et l'affirmation de la peinture qui s'y exprime se décident toutefois à travers un débat avec les constructivistes autour de la question du rôle des nouvelles techniques dans la production artistique. Dans ce qui suit, je vais d'abord rappeler le sens critique et phénoménologique qu'a reçu le "Carré noir» comme ouverture au suprématisme et à l'art abstrait au $\mathrm{XX}^{\mathrm{e}}$ siècle avant d'examiner le sens du retour à la figuration dans les dernières œuvres de Malévitch.

\section{Malévitch comme « Kulturkritiker »}

Malévitch n'est certes pas le premier pour qui la recherche picturale est une recherche philosophique. Il s'agit pour lui aussi de la vérité en peinture : «En art on a besoin de Vérité et non de sincérité », écrit-il dans «Du cubisme et du futurisme au suprématisme. Le nouveau réalisme pictural » $(1916)^{1}$. Mais, à l'encontre de Paul Cézanne, qui fonde cette recherche sur une analyse de la perception visuelle, ou bien encore à l'encontre aussi de Giorgio de Chirico, qui reconstruit l'espace pictural selon une recherche métaphysique de la réalité des objets, le peintre russe recherche cette vérité en révélant les conditions de la création artistique de l'image picturale. Cette recherche l'entraîne dans un mouvement radical abandonnant et éliminant l'une après l'autre les différentes conventions de l'image picturale: la dépendance à l'égard de l'objet représenté, la construction de l'espace selon les règles de la perspective ou selon les règles du cubisme, la ligne horizontale, l'espace de la

\footnotetext{
${ }^{1}$ Kazimir Malévitch, «Du Cubisme et du futurisme au suprématisme. Le nouveau réalisme pictural » (1916), dans Id., De Cézanne au suprématisme. Tous les traités de 1915 à 1922, trad. fr. J.-C. et V. Marcadé avec la collaboration de V. Schiltz, préface et présentation de J.-C. Marcadé, Lausanne, Éditions de L'Âge d'Homme, 1974, p. 49.
} 
représentation. Le parcours de Malévitch possède ceci de particulier qu'un demi-siècle avant cet événement qui fait aboutir l'art plastique dans la philosophie, selon l'expression d'Arthur Danto, Malévitch l'a déjà réalisé en abandonnant effectivement son métier de peintre pour s'adonner pendant quelques années à la philosophie et à la rédaction de son livre, Suprematismus - Die gegenstandslose Welt ${ }^{1}$. Or, cet aboutissement de la peinture en philosophie se produit pour des raisons exactement opposées à celles mentionnées par Danto : tandis que, pour celui-ci, la fin de l'art résulte du fait que les techniques de la production artistique ont tellement évolué qu'il devient enfin possible de créer une œuvre d'art - le «Brillo Box » de Warhol - dont les qualités visuelles sont identiques avec les qualités de l'objet dans le monde dont elle est une copie ${ }^{2}$, Malévitch rencontre la philosophie sur son chemin suprématiste en éliminant toute référence à l'évidence d'un objet déjà donné. En creusant et en approfondissant les conditions de la création artistique, il s'applique à révéler et à réfléchir dans l'espace pictural une dimension que présuppose toute apparition d'objet.

Pour Malévitch, le suprématisme n'est pas seulement la découverte du formalisme, même s'il considère la réduction à la forme comme le point zéro de toute création artistique. Pour lui, le suprématisme ne consiste pas en un courant artistique parmi d'autres et ne se limite pas non plus à réaliser une nouvelle approche de la création artistique. Le suprématisme est davantage une ontologie invitant à une nouvelle entente de la réalité, de l'homme et du monde, de la création artistique et de l'espace pictural ${ }^{3}$. Quoiqu'on pense de la qualité philosophique des écrits de Malévitch, plusieurs interprètes ont vu dans la radicalité avec laquelle il met en question toute objectivité en peinture, pour s'approcher de ce qui conditionne l'apparaître pictural en tant

1 Kazimir Malévitch, Suprematismus - Die gegenstandslose Welt, Werner Haftmann (Hrsg.), Köln, DuMont Buchverlag, 1989.

2 Arthur C. Danto, Beyond the Brillo Box. The Visual Arts in Post-Historical Perspective, Berkeley/Los Angeles/London, University of California Press, 1992, en particulier « The Artworld Revisited: Comedies of Similarity », p. 33-53.

${ }^{3}$ « Aufgabe der Kunst war es, Erregungs- oder Spannungszustände in die Wirklichkeit zu verkörpern, in eine "Wirklichkeit" die doch immer nur Kulisse bleibt. Die wahre Wirklichkeit bleibt unsichtbar, denn es kann die gegenständliche Kunst nicht in sie eindringen. [...] Das Können, etwas Vorhandenes nachzubilden, war bisher der Genius der Kunst. Die Neue Kunst hingegen hat diesem Genius entsagt und versucht, die Erregung sichtbar zu machen. Ihr Genius ist nicht mehr die Kunst des Nachahmens, sondern der Verkörperung der Erregung als Erweckung zum Sein, zur Wirklichkeit. Als Wirklichkeit tritt die Kunst in ihre Suprematie» (Kazimir Malévitch, Suprematismus - Die gegenstandslose Welt, op. cit., p. 180). 
que tel, une expression d'une recherche phénoménologique ${ }^{1}$. Emmanuel Martineau, l'auteur de Malévitch et la philosophie, y voit même une expression avant la lettre de la différence ontologique qui est l'ouverture de la philosophie heideggérienne ${ }^{2}$. C'est le rapport à l'être de la création artistique qui détermine la recherche de Malévitch et qui contient l'exigence de libérer la peinture de toute dépendance à l'égard de l'étant (Vorhandenes ce terme est déjà employé par Malévitch), fut-ce l'étant du phénomène. Il y a dans la recherche de Malévitch une conscience très développée de ce que les définitions traditionnelles de la création artistique, qui ont expliqué celle-ci en la soumettant à une réalité déjà donnée, obstruent l'accès à la véritable réalité. Cette conscience est à la mesure de la radicalité avec laquelle il rejette tout rapport à l'étant afin de pouvoir présenter l'invisible qui conditionne la création artistique. Selon Martineau, le peintre russe va, dans sa recherche, plus loin que Heidegger, au point de l'avoir déjà déconstruit. En effet, alors que celui-ci explique la notion de "phénomène » (Erscheinung) en l'attribuant à ce qui se montre de soi-même, celui-là abandonne cette notion à cause du lien qu'elle conserve avec l'apparition d'un objet. N'est phénomène que ce qui se montre comme étant. Or, Malévitch cherche à éliminer toute référence à un étant afin d'avoir accès à l'apparaître pur. Le néant n'aura pas le même sens dans les écrits de Malevitch que dans l'ontologie existentiale de l'historialité chez Heidegger ${ }^{3}$.

Quoiqu'il en soit, Malévitch peut, par son approche spécifique, affirmer à la fois l'autonomie irréductible de la création artistique et l'identité entre l'art et la vie. L'autonomie irréductible de la création artistique signifie que l'art produit ses formes à partir de sa propre condition et ne les emprunte plus ni à la culture ni à la nature : « Il y a création seulement là où dans les tableaux apparaît la forme qui ne prend rien de ce qui a été créé dans la nature, mais qui découle des masses picturales, sans répéter et sans modifier

${ }^{1} C f$. Felix Philipp Ingold, «Welt und Bild. Zur Begründung der suprematistischen Ästhetik bei Kazimir Malevič », art. cit., p. 375. L'auteur parle en terme d'une «InErscheinung-treten-lassen der Phänomene » par quoi le caractère pictural de l'image (die Bildlichkeit des Bildes) devient visible.

${ }^{2}$ Emmanuel Martineau, Malévitch et la philosophie. La question de la peinture abstraite, Lausanne, Éditions de L'Âge d'Homme, 1977. Voir également JeanClaude Marcadé, « Le Suprématisme de K.S. Malevič ou l'art comme la réalisation de la vie », dans Revue des études slaves, 56/1, 1984, p. 66 : « Il s'agit d'une libération du regard en direction de l'être par la mise entre parenthèses de l'étant, selon une formulation de Heidegger $\gg$.

${ }^{3}$ Emmanuel Martineau, Malévitch et la philosophie. La question de la peinture abstraite, op. cit., p. 49 et $191 \mathrm{sq}$. 
les formes premières des objets de la nature $»^{1}$. Le monde sans objet doit être produit par la création artistique. C'est ainsi qu'elle se frayerait un chemin vers la vraie réalité qui conditionne et la culture et la nature. Cette réalité, Malévitch l'appelle « excitation » (Erregung) ou «tension» (Spannung). Il ne s'agit pas de représenter la tension entre les forces ou les conflits dans le monde ou dans la nature, mais la tension inhérente à la sensibilité pure. Ainsi, ce que l'œuvre d'art présente, ce n'est pas tant une image de la réalité que la condition d'être de la réalité : l'événement des tensions en mouvement permanent ${ }^{2}$.

Cette façon de présenter la réalité distingue ce que Malévitch appelle «le tableau vivant» et "les formes vivantes» de la représentation caractéristique du tableau traditionnel ${ }^{3}$. Tandis que celui-ci, en copiant les formes de la nature, fixe tout vivant dans l'espace de la toile et le transforme ainsi «en un état d'immobilité morte», dans celui-là, "[d]ans l'art du suprématisme[,] les formes vont vivre ainsi que toutes les formes de la nature ${ }^{4}$. « De telles formes ne seront pas la répétition des objets qui vivent dans la vie, mais seront-elles-mêmes un objet vivant. La surface coloriée est la forme vivante réelle » ${ }^{5}$. Ce tableau « vivant » n'est plus bidimensionnel ni tridimensionnel, mais transforme l'espace pictural en une nouvelle «quatrième» - dimension, celle d'un espace-mouvement présentant la condition et le principe de la création artistique. La création artistique qui réussit cette percée non seulement libère l'art, mais aussi l'homme de leur soumission à l'objectivation. L'art ne doit plus se mesurer à la nature, parce qu'il a abandonné le principe de l'imitation et rejoint lui-même les forces et les tensions de la nature. L'homme ne doit plus se mesurer à un monde qui le réduit à un instrument parce que le monde se révèle être par la création artistique, sans objet et sans objectivation, pur rythme de la sensibilité où l'homme rejoint sa véritable nature.

\footnotetext{
${ }^{1}$ Kazimir Malévitch, «Du Cubisme au suprématisme en art, au nouveau réalisme de la peinture en tant que création absolue» (1915), dans Id., De Cézanne au suprématisme. Tous les traités de 1915 à 1922, op. cit., p. 38.

2 Jean-Claude Marcadé parle pour cette raison d'une «vision radicalement moniste du monde » chez Malévitch et de l'«identification de l'acte pictural et du mouvement du monde » ( $c f$. Jean-Claude Marcadé, «Le Suprématisme de K.S. Malevič ou l'art comme la réalisation de la vie », art. cit., p. 64).

${ }^{3}$ Kazimir Malévitch, «Du Cubisme et du futurisme au suprématisme. Le nouveau réalisme pictural» (1916), art. cit., p. 55 sq.

${ }^{4}$ Ibid., p. 67.

${ }^{5}$ Ibid., p. 63.
} 
C'est dans le suprématisme blanc que se réalise cette transformation. L'évolution du «Carré noir» qui ouvre le suprématisme au «Blanc sur blanc » qui l'achève (1918) est significative pour le sens même du suprématisme. L'effacement progressif de l'opposition entre noir et blanc au profit de la seule présence du blanc sur blanc n'accuse pas seulement le mysticisme dans la recherche de Malévitch, mais est consécutif à l'ouverture du suprématisme et annonce déjà la rupture avec le constructivisme. La couleur noire reste liée à la ligne qui est traditionnellement utilisée pour dessiner les contours et circonscrire l'objet à peindre. C'est pourquoi elle est caractéristique de la formation graphique et de l'écriture qui gardent, toutes deux, un rapport aux contours, à la délimitation et à l'identification de l'objet. Érigée en surface par la forme du carré, la couleur noire nie cette fonction de la ligne et rend par là la visibilité de l'image-objet opaque, mais elle ne permet pas de créer et de transformer l'espace pictural en un espace mouvement. En tant que négation de toute possibilité, elle est trop massive et trop statique. Le blanc, en revanche, est dynamique et potentialité pure, évoquant à la fois le repos imperturbable et le mouvement infini des possibilités ${ }^{1}$.

Quel sens de la modernité se réfléchit dans le suprématisme de Malévitch ? Il va sans dire que la recherche picturale de l'être du monde en dehors de toute objectivation implique une critique radicale de la rationalité occidentale. Dans la formulation de cette critique, Malévitch rejoint les attaques aux principes épistémologiques de cette rationalité exprimées dans la philosophie de Friedrich Nietzsche. C'est à travers la recherche de la création artistique que sont récusées et abandonnées l'une après l'autre les dichotomies classiques de l'être et de l'apparaître, de l'opposition entre sujet et objet, de la compréhension du monde comme totalité ou connexion des objets. C'est à travers la recherche de la création artistique qu'un accès est forgé vers ce qui conditionne la conscience de la réalité et ce qui reste invisible dans la rationalité objectivant l'homme et le monde, à savoir le rythme et l'excitation de l'apparaître pur ou de la sensibilité pure en-deçà de toute objectivation. Il est intéressant de constater que Malévitch rejoint de cette façon dans sa recherche picturale l'analyse que Maurice Merleau-Ponty a donnée de la perception cinématographique, ainsi par exemple lorsque celui-ci écrit : «Bien loin d'être un simple "déplacement”, le mouvement est

${ }^{1}$ « Die Bewegung des Suprematismus geht bereits in dieser Richtung, sie ist auf dem Wege zur weißen gegenstandslosen Natur, zu weißen Erregungen, zum weißen Bewußtsein und zu weißer Reinheit als der höchsten Stufe jeden Zustandes, der Ruhe wie der Bewegung » (Kazimir Malévitch, Suprematismus - Die gegenstandslose Welt, op. cit., p. 181). 
inscrit dans la texture des figures ou des qualités, il est comme révélateur de leur être ${ }^{1}$. Dépassant la distinction entre le représenté et la présentation dans l'espace pictural, transformant celui-ci en un espace-mouvement et le révélant en tant que tel comme mouvement de l'être, Malévitch nous introduit à une nouvelle vision du monde - qui n'est pas donnée comme un objet en dehors ou au-delà de la création artistique — et de l'image — qui n'est pas une représentation. Cette nouvelle vision a également son point de départ, comme Mauro Carbone le décrit à propos de Merleau-Ponty dans une réflexion sur l'image cinématographique, dans

une réhabilitation ontologique de la surface sur laquelle l'apparaître se montre; une surface qu'il ne faut, dès lors, plus penser comme le voile qui cacherait le vrai et qui devrait donc être levé ou même percé, mais comme l'écran qui s'avère être la condition décisive pour faire voir les images où la vérité se manifeste ${ }^{2}$.

Il semble clair, dès lors, que le tournant vers le suprématisme et le mysticisme auquel aboutit celui-ci avec «Blanc sur blanc» impliquent un rejet de la production de masse industrielle et du naturalisme inhérent au matérialisme théorique des sciences positives dans le monde moderne. Pourtant, ce rapport est plus complexe et plus ambigu. La percée du suprématisme, Malévitch l'énonce explicitement en continuité avec les élans et les acquis du futurisme et du cubisme. Le premier croquis d'un carré à moitié noir apparaît dans l'opéra futuriste de Matjushin "Victoire sur le soleil », pour lequel Malévitch avait dessiné les costumes et la scène. Il s'agit d'un véritable optimisme à l'égard de la technique qui va libérer l'homme de sa condition terrestre donnant naissance au Kraftmensch capable de soumettre à son pouvoir les énergies de la nature. Le carré noir y figure comme un soleil obscurci qui implique la destruction de toutes les notions-clés par lesquelles l'homme s'est interprété lui-même, la nature et le monde, à commencer par l'héritage platonicien, le soleil étant entendu comme métaphore du bien qui soumet la création artistique à la définition d'imitation.

Dans une lettre à Matjuschin de 1915, il s'avère que Malévitch était très conscient de la signification du carré noir dans cet opéra et pour l'évolution de son œuvre : il y appelle la fonction du carré noir «l'embryon

${ }^{1}$ Maurice Merleau-Ponty, Résumés de cours. Collège de France 1952-1960, Paris, Éditions Gallimard, 1968, p. 15.

${ }^{2}$ Mauro Carbone, «Pour une réhabilitation ontologique de l'écran. Merleau-Ponty et Lyotard entre peinture et cinéma », dans Id., L'Empreinte du visuel. Merleau-Ponty et les images aujourd'hui, Genève, Éditions MetisPresses, 2013, p. 127-141. 
de toutes les possibilités, qui, dans son développement, devient une force terrible ; sa scission engendre dans la peinture une culture prodigieuse. Dans l'opéra, il signifie le début de la victoire $»^{1}$. Il s'ensuit que, pour Malévitch, la découverte du carré noir et celle de sa signification artistique n'impliquent nullement un rejet des forces de transformation de la modernité, mais elles en découlent au contraire. C'est grâce à la destruction des formes héritées de la culture du passé - destruction qui est inhérente aux nouvelles forces de production - que la recherche d'un nouvel espace pictural devient recherche philosophique. «La peinture a depuis longtemps fait son temps et le peintre lui-même est un préjugé du passé »², affirme Malévitch dans un texte sur le suprématisme datant de 1920. Cet abandon de la peinture, ce n'est pas lui qui le proclame, mais c'est la transformation du monde moderne qui a dérobé à la peinture son évidence et son effectivité. Or, cette destruction signifie aussi une chance : la chance de s'interroger sur la condition de la création artistique et de libérer l'espace pictural de sa soumission à ce qui lui reste extérieur. Il y a donc bel et bien enjeu épistémologique, crise du savoir et dépassement du rapport entre sujet et objet dans la recherche picturale de Malévitch, mais celle-ci ne met pas encore en question l'idée du progrès réalisé par l'effectivité des nouvelles technologies.

\section{Le retour au figuratif}

Il est indéniable que le retour au figuratif dans l'espace pictural rompt avec la recherche du « sans objet » qui a défini l'enjeu même du suprématisme. Mais ce retour signifie-t-il inévitablement un retour en-deçà de la création artistique caractéristique du suprématisme et un désaveu de sa philosophie ? Ou bien invite-t-il à une recherche supplémentaire et à un approfondissement de sa signification après les réalisations et les aboutissements de l'espace pictural suprématiste ? Malévitch continue à signer ses tableaux par un carré noir. Le choix et la disposition des couleurs, la réduction de la forme, et même - jusqu'à un certain degré - la construction de l'espace pictural continuent à se référer au style et à l'œuvre artistique de Malévitch. Qu'est-

${ }^{1}$ «stellt ein schwarzes Quadrat dar, den Keim aller Möglichkeiten, der in seiner Entwicklung zu fürchterlicher Kraft anwächst. Er ist der Urahn des Würfels und der Kugel ; seine Spaltung bringt in der Malerei eine erstaunliche Kultur hervor. In der Oper bezeichnet er den Beginn des Sieges » (Evelyn Weiss (Hrsg.), Kazimir Malewitch. Werk und Wirkung, Köln, DuMont, 1995, p. 119).

${ }^{2}$ Kazimir Malévitch, «Le Suprématisme, 34 dessins » (1920), dans Id., De Cézanne au suprématisme. Tous les traités de 1915 à 1922, op. cit., p. 123. 
ce qui est alors recherché par la réintroduction de la figuration ? Et comment celle-ci modifie-t-elle l'espace pictural ?

Pour pouvoir répondre à ces questions, il importe de se rappeler brièvement les raisons du conflit avec les constructivistes ${ }^{1}$. Entre les suprématistes et les constructivistes, il y a un fond commun : plusieurs constructivistes déclarés, tel Rodchenko, furent élèves de Malévitch qui, à son tour, devint membre du groupe d'artistes INChUK fondé par Rodchenko et Kandinsky en 1920. Ils partagent la recherche du sans-objet, la réduction à la forme et la conviction révolutionnaire d'un art au service du réalisme social. Deux éléments sont toutefois décisifs pour comprendre la rupture du constructivisme avec le suprématisme : ils concernent le rapport entre la création artistique et le rôle des nouvelles techniques. Sous l'influence des counterreliefs de Vladimir Tatlin, des constructions matérielles disposées de telle sorte que les rapports de l'espace réel sont transformés, les constructivistes abandonnent l'espace pictural au profit de la construction des objets réels et des interventions dans l'espace réel. Ils se rallient en outre à la production industrielle des formes artistiques, y voyant un moyen pour transformer la société selon les principes de la création artistique. C'est dans cette perspective que, pour eux, l'art se réalise dans la vie.

Malévitch n'a jamais accompli ce passage à la construction d'un objet réel, même si ce pas semble logique après l'achèvement de la forme suprématiste dans l'espace pictural de «Blanc sur blanc ». La construction de l'espace suprématiste n'est-elle pas la mise en rapport des éléments matériels afin de créer un effet de force et de tension? Malévitch n'avait-il pas luimême déclaré que la peinture était un préjugé du passé ? Il n'approuve pas non plus la soumission de la création artistique à la production industrielle même si, lui aussi, prévoit la transformation de la société par les formes suprématistes $^{2}$. Le problème qu'il rencontre dans les discussions avec les constructivistes, c'est le principe de base du tournant suprématiste, à savoir l'autonomie de la création artistique. L'affirmation de ce principe a des conséquences pour définir le rapport entre la création artistique et la production technique. L'autonomie signifie que la recherche picturale pos-

${ }^{1}$ Tatjana Gorjatschewa, « Suprematismus und Konstruktivismus. Antagonismus und Ähnlichleit, Polemik und Zusammenarbeit», dans Staatliche Kunsthalle BadenBaden, Von der Fläche zum Raum. Malewitsch und die frühe Moderne, Köln, Walter König, 2008, p. 16-28.

${ }^{2}$ " "Alle Dinge, unsere ganze Welt sollte sich in suprematistische Formen kleiden, das heißt Stoffe, Tapeten, Töpfe, Teller, Möbel, Schilder, mit einem Wort, alles sollte mit suprematistischen Zeichnungen als der neuen Form der Harmonie versehen sei”, erklärten die UNOWIS-Anhänger » (ibid., p. 20). 
sède une finalité en soi. Seule cette finalité en soi sauvegarde la possibilité de réaliser une réflexion sur la condition de la création artistique et de l'œuvre d'art. Dans la production industrielle, cette possibilité disparaît : l'art devient utilitaire, c'est-à-dire la création artistique se met au service des développements techniques et la production de formes nouvelles répond à l'exigence d'une formation (politique et idéologique) des pratiques et des besoins sociaux. Bref, l'artiste devient ingénieur et l'œuvre d'art design.

Dans ce contexte, le retour au figuratif signifie donc en premier lieu et de façon paradoxale l'affirmation de l'espace pictural en tant qu'expression de l'autonomie de la création artistique. Cette affirmation explique d'ailleurs l'héritage inégal du constructivisme et du suprématisme dans l'esthétique communiste de l'URSS. Tandis que Rodchenko se tourne vers la production photographique et se met par là dans des contextes historiques fort problématiques (Staline commence une politique de «purification » à partir de 1927, aboutissant également dans l'émergence des camps de travail) au service de la propagande communiste, Malévitch sauvegarde un espace de réflexion qui lui permet de garder distance, mais qui le rend aussi suspect aux yeux des autorités. Le retour au figuratif, entendu comme affirmation de l'espace pictural, peut donc être interprété en continuité avec le même principe que Malévitch avait invoqué pour réaliser la percée du suprématisme. Il s'ensuit qu'il est possible de dire que le retour à l'image picturale engage aussi une nouvelle recherche sur la condition de l'être de la création artistique et de l'œuvre d'art. Ce qui a changé dans cette recherche se définit par la réintroduction du figuratif dans une création artistique qui a rendu visible le monde sans objet. Comment est-il possible d'articuler cette modification?

Ce n'est pas une figure quelconque qui est représentée, c'est la figure humaine. Certes, d'autres objets sont également identifiables dans le tableau : maison, avion en l'air, horizon, chevaux, mais ceux-ci sont à l'arrière-plan et semblent emprunter leur signification à la présence de la première figure qui domine l'espace pictural. Cette présence, même abstraite, n'est pas tout à fait sans qualités : elle est nommée et reconnaissable comme "paysan(s)» ou « homme avec chemise jaune » ou « figure(s) féminine(s) », souvent en rapport avec un paysage où elle apparaît en même temps comme à distance, comme égarée ou disloquée. C'est cette présence qui est affirmée comme condition de l'être de la création artistique à un moment où Staline avait lancé ses plans quinquennaux soumettant les paysans à un processus de collectivisation agricole intégrale et imposant à la société soviétique une transformation rapide par l'industrialisation.

Ce n'est pas non plus une technique de présentation quelconque. Les techniques de la peinture suprématiste ne sont pas rejetées : on peut remar- 
quer la réduction de la forme (la figure humaine est comme effacée, elle n'est pas identifiable comme une personne vivante, à l'exception près de quelques portraits et autoportraits que Malévitch a peints à la même époque), la matérialité de la couleur (le blanc comme couleur de base, la dominance de l'opposition entre noir et blanc qui réapparaît dans le visage de la figure), le mouvement et la tension de l'espace pictural (la dissolution de la couleur réapparaît à l'intérieur de la figure). C'est donc la seule introduction de la figure humaine qui transforme l'espace pictural. Elle affirme en premier lieu une limitation. La sensibilité, que Malévitch avait mise au centre de sa recherche suprématiste, ne relève plus d'une tension évoquée par une dimension spatiale anonyme, mais s'inscrit dans les limites d'une subjectivité. Elle réintroduit en outre une division entre figure et monde. L'introduction de la figure humaine semble dès lors récuser la recherche de la sensibilité pure et l'équation entre la vérité de l'être et la sensibilité pure - qui était l'aboutissement de la peinture suprématiste.

$\mathrm{Du}$ coup, le sens et la fonction de la couleur et du mouvement changent dans l'espace pictural. La couleur se lie davantage au mouvement par un procédé que Malévitch avait déjà appliqué lors de la période suprématiste en ajoutant différentes nuances de blanc, évoquant ainsi un mouvement de disparition ou de dissolution, mais ce procédé est réinvesti par une nouvelle façon de disposer les couleurs gardant un lien visible avec les touches du pinceau. Le mouvement n'apparaît plus comme expression de l'espace du tableau, mais à partir de la distinction entre le mouvement d'un monde qui demeure indéfini - même s'il s'avère par la présence des figures et la division des champs de couleurs plus proche de la culture que de la nature - et le mouvement apparaissant à partir de la figure humaine. Le rapport entre les deux déstabilise l'espace pictural. Il en résulte que la tension se crée à l'intérieur de la figure et à la fois dans le rapport entre la figure et l'espace d'un monde - rapport qui surprend, effraie et apparaît même parfois menaçant, ce qui est accentué par la disposition des couleurs noires et violentes. Le sens même de la couleur se trouve ainsi transformé, car elle cherche davantage à solliciter l'émotion du spectateur au lieu d'évoquer le mouvement caractéristique de l'espace pictural suprématiste (la tension d'un apparaître/disparaître d'une forme pure). Pour toutes ces raisons, il me semble que le retour au figuratif a encore pour but de révéler la condition du monde comme «tension» et "mouvement», mais y ajoute le sens de la fragilité et de l'irréductibilité de la condition humaine : exposition au monde qui conditionne l'apparaître du monde.

Quel sens de la modernité apparaît à partir de ce retour au figuratif ? Il est clair que le statut de la réflexion réalisée dans l'espace pictural s'est 
modifié à partir de ce retour. En fait, c'est la conception même de l'autonomie irréductible de la création artistique et l'affirmation consécutive de l'identité entre l'art et la vie qui se trouvent transformées. L'idée de l'autonomie n'est pas abandonnée. Bien au contraire: l'espace pictural est de nouveau affirmé comme un espace propre où peuvent être recherchées, examinées et révélées les conditions de l'apparaître et de la création artistique. Le tournant suprématiste est dans ce sens-là irréversible : désormais, la création artistique ne peut acquérir et sauvegarder son autonomie qu'en réfléchissant sur la condition de toute production de l'apparaître. Le retour au figuratif n'implique donc pas nécessairement une rupture avec l'exigence suprématiste de libérer l'image de sa soumission à tout ordre d'objectivation. Seulement, il s'avère que l'affirmation de l'autonomie de la création artistique ne suffit pas à révéler, à partir des seuls moyens de la peinture, la véritable réalité qui conditionne le monde - et donc à révéler ainsi l'identité entre l'art et la vie. Au moins la figure humaine et son rapport au monde, tels qu'ils sont exposés à l'objectivation par le processus d'industrialisation et de collectivisation agricole dans le socialisme soviétique, résistent à une telle réduction et nécessitent de corriger l'entente suprématiste de l'espace pictural. Il en résulte que la réaffirmation de l'autonomie de la création artistique va de pair avec une prise de conscience de la séparation entre l'art et la vie.

Il est possible de préciser ce nouvel espace de réflexion à partir des oppositions suivantes. Il y a tout d'abord l'opposition entre création artistique et production industrielle. Sans leur distinction, on ne peut pas voir comment l'espace pictural peut affirmer un rapport à la vérité. La création artistique qui se subordonne à la production industrielle, tout en se réalisant dans la société par la reproduction technique de masse, devient utilitaire : elle peut bien embellir à grande échelle la vie quotidienne et transformer la vie pratique, mais elle n'a plus le pouvoir de réfléchir et de révéler la condition du monde et de libérer ainsi l'art des systèmes d'objectivations qui lui sont inhérents. L'acte individuel de la création artistique (avec ses choix et ses délibérations - mêmes inconscients) est exigé pour pouvoir récuser l'évidence d'un monde déjà donné. Il y a ensuite l'opposition entre l'autonomie de l'espace pictural et sa dépendance à l'égard du représenté. Cette distinction semble restée en-deçà de la percée suprématiste qui transforme l'espace pictural en un espace-mouvement. Or, le retour de la figuration n'implique pas nécessairement une rupture avec cette percée : il atteste seulement d'une prise de conscience de la complexité des rapports au monde, évoquant le mouvement non plus à partir d'une vision abstraite d'une forme indéfinie en voie de disparition, mais à partir d'une vision qui a son origine dans les 
limites de la figure humaine. Seule cette dépendance à l'égard de la figure humaine permet à l'espace pictural qui possède le pouvoir de révéler la condition du monde de tenir compte aussi d'une position dans le monde et d'un engagement dans la vie. Enfin, il faut opposer le monde et la figure humaine. Ce que les derniers tableaux de Malévitch révèlent, c'est qu'il est tout autant impossible de faire rejoindre l'homme et le monde que de faire dissoudre leur rapport. Si la figure humaine est nécessaire comme condition de l'accès au monde, l'espace pictural révèle dans sa recherche de l'être du monde la fragilité de cette condition. Tout en introduisant la figure humaine comme forme primaire, il fait voir le monde comme une transcendance irréductible qui dépasse sans cesse la figure et qui la met dans une position où elle n'est pas à sa place.

La réaffirmation de l'espace pictural dans un contexte où le passage à la production industrielle des objets s'impose, et où ce passage semble achever certains développements de la recherche de l'espace pictural, est clairement une prise de position à l'égard de ce passage et de l'abandon de la peinture qui en résulte. Dès lors, il est possible de reformuler et de préciser le sens critique de la création artistique exprimée par cette affirmation. Il est exclusivement lié à une réhabilitation ontologique de l'espace pictural et s'énonce tout en tenant compte d'une nouvelle entente de cet espace. Il concerne à la fois (1) le rôle de la production technique dans la création artistique : ne pas trahir l'autonomie de l'espace pictural, c'est-à-dire veiller à le créer comme un espace de réflexion; (2) la tâche de l'artiste dans le monde moderne : chercher la vérité dans la création artistique, c'est-à-dire ne pas soumettre celle-ci à des systèmes d'objectivation dans le monde ; (3) la recherche de l'espace pictural : faire voir les conditions de l'apparaître du monde, y compris sa position dans le monde.

\section{En guise de conclusion}

Le dernier Malévitch délimite donc une position à part dans le débat sur la question de la représentation. Il est l'un des premiers à avoir percé et abandonné les présupposés métaphysiques de la conception traditionnelle de la représentation dans sa recherche de faire apparaître la condition du monde et de l'espace de la représentation. Mais il est aussi l'un des premiers à avoir réintroduit le figuratif dans l'espace pictural après cet abandon. Ce retour ne signifie pas nécessairement une rupture avec les acquis de la recherche suprématiste mais - et c'est ce que nous avons cherché à montrer dans ce qui précède - il s'exprime par une même inspiration et en continuité avec 
l'espace réflexif ouvert par le tournant suprématiste. Dans le retour au figuratif, il s'agit en effet de sauvegarder l'autonomie de la création artistique face au passage à la fabrication industrielle d'objets et d'affirmer tout à la fois l'idée d'un espace pictural comme espace de réflexion et de distance critique à l'égard du monde présent.

Le silence qui frappe la dernière période de Malévitch est donc mal placé et risque même de recéler une cécité à l'égard des pouvoirs critiques et phénoménologiques de l'espace pictural dont les conséquences se prolongent hélas jusqu'à nos jours. Le terme de "nostalgique » par lequel Lyotard qualifie le suprématisme de Malevitch passe sous silence un pouvoir d'invention qui est capable de poser à nouveau, après avoir détruit les réponses données, la question de la représentation, c'est-à-dire la question de l'image et de son rapport au monde, et de lancer une nouvelle recherche de la création artistique picturale. Aussi la dernière période de Malévitch révèle-telle qu'il est erroné de considérer la question de la représentation - après son émancipation des contraintes de la correspondance avec un monde ou un modèle donnés - arbitraire et dépassée dans le sens où tout devient également représentable, comme dit Jacques Rancière dans Le Destin des images, caractérisant ainsi le régime de l'art qu'il appelle esthétique ${ }^{1}$. Il faut plutôt affirmer le contraire, à savoir que la question du choix des contraintes par lesquelles les moyens de la représentation sont réinvestis dans un espace pictural devient un enjeu difficile et d'autant plus important que c'est de ce choix que dépend la force et la résistance critiques de l'œuvre d'art à l'égard du présent.

${ }^{1}$ « Tout est à égalité, également représentable. Et cet "également représentable" est la ruine du système représentatif » (Jacques Rancière, Le Destin des images, Paris, Éditions La fabrique, 2003, p. 136). 